\title{
LIOUVILLE TYPE THEOREM FOR HIGHER ORDER HARDY-HÉNON SYSTEM OF INEQUALITIES
}

\author{
LI-HUA MIN
}

Abstract. In this paper, we prove some new Liouville type theorems for fourth order and more general higher order Hardy-Hénon systems of inequalities. The test function method is applied to show the nonexistence of nontrivial nonnegative global solutions, which improve and extend some recent results.

Mathematics subject classification (2010): 35K41, 35B53, 35B08.

Keywords and phrases: Higher order, Liouville type theorem, system of inequalities, test function method.

\section{REFERENCES}

[1] S. N. Amstrong And B. SIRAKOV, Nonexistence of positive supersolutions of elliptic equations via the maximum principle, Comm. Partial Differential Equations, 36, 11 (2011), 2011-2047.

[2] M. F. Bidaut-VÉRON And H. Giacomini, A new dynamical approach of Emden-fowler equations and systems, Adv. Differential Equations, 15, 11-12 (2010), 1033-1082.

[3] M. CALANCHI AND B. RuF, Radial and non radial solutions for Hardy-Hénon type elliptic systems, Calc. Var., 38, (2010), 111-133.

[4] C. CowAn, A Liouville theorem for a fourth order Hénon equation, http: //arxiv .org/pdf/1110. 2246.pdf, (2011).

[5] L. Dupaigne And A. C. Ponce, Singularities of positive supersolutions in elliptic PDEs, Selecta Math. (N.S.), 10, 3 (2004), 341-358.

[6] Y. V. Egorov, V. A. Galaktionov, V. A. Kondratiev and S. Pohozaev, On the necessary conditions of global existence to a quasilinear inequality in the half-space, C. R. Acad. Sci. Paris Sér. I. Math., 330, (2000), 93-98.

[7] M. Escobedo AND M. A. HERRERo, Boundedness and blow up for a semilinear reaction-diffusion system, J. Differential Equations, 89, 1 (1991), 176-202.

[8] M. Fazly and N. Ghoussoub, On the Hénon-Lane-Emden conjecture, http: //arxiv .org/pdf / 1107.5611.pdf, (2011).

[9] J. FÖLDES, Liouville theorems, a priori estimates, and blow-up rates for solutions of indefinite superlinear parabolic problems, Czechoslovak Math. J., 61, 136 (2011), 169-198.

[10] V. A. Galaktionov And S. I. Pohozaev, Existence and blow-up for higher-order semilinear parabolic equations: Majorizing order-preserving operators, Indiana Univ. Math. J., 51, 6 (2002), 1321-1338.

[11] B. GIDAS AND J. SPRUCK, Global and local behavior of positive solutions of nonlinear elliptic equations, Comm. Pure Appl. Math., 34, 4 (1981), 525-598.

[12] Z. X. JiAng AND S. N. ZHENG, Fujita-Liouville type theorem for coupled fourth-order parabolic inequalities, Chin. Ann. Math., 33B, 1 (2012), 107-112.

[13] A. G. Kartsatos And V. V. KuRTA, On a Liouville-type theorem and the Fujita blow-up phenomenon, Proc. Amer. Math. Soc., 132, (2004), 807-813.

[14] L. H. Min, X. P. YANG AND C. F. GUI, Entropy estimates and large time behavior of solutions to a fourth order nonlinear degenerate equation, Commun. Contemp. Math., (2013), DOI: 10.1142/S0219199712500666. 
[15] L. H. Min AND X. P. YANG, Finite speed of propagation and algebraic time decay of solutions to a generalized thin film equation, Submitted to Communications on Pure and Applied Analysis (CPAA).

[16] E. MitidieRI, Nonexistence of positive solutions of semilinear elliptic systems in $\mathbb{R}^{N}$, Differential Integral Equations, 9, 3 (1996), 465-479.

[17] E. Mitidieri AND S. I. PohozAev, A priori estimates and the absence of solutions of nonlinear partial differential equations and inequalities, Tr. Mat. Inst. Steklova, 234, (2001), 1-384.

[18] Q. H. PHAN, Liouville-type theorems and bounds of solutions for Hardy-Hénon elliptic systems, Adv. Differential Equations, 17, 7-8 (2012), 605-634.

[19] Q. H. Phan And Ph. Souplet, Liouville-type theorems and bounds of solutions of Hardy-Hénon equations, J. Differential Equations, 252, (2012), 2544-2562.

[20] P. PoláčiK, P. QuitTner AND PH. SouPlet, Singularity and decay estimates in superlinear problems via Liouville-type theorems, Part I: Elliptic equations and systems, Duke Math. J., 139, (2007), 555-579.

[21] P. PolÁčIK, P. QuitTner And Ph. SouPlet, Singularity and decay estimates in superlinear problems via Liouville-type theorems, Part II: Parabolic equations, Indiana Univ. Math. J., 56, (2007), 879-908.

[22] PH. Souplet, The proof of the Lane-Emden conjecture in four space dimensions, Adv. Math., 221, (2009), 1409-1427.

[23] X. W. XU, Uniqueness theorem for the entire positive solutions of biharmonic equations in $\mathbb{R}^{n}$, Proc. Roy. Soc. Edinburgh Sect. A, 130, 3 (2000), 651-670.

[24] X. D. YAn, A Liouville-type theorem for higher order elliptic systems, J. Math. Anal. Appl., 387, (2012), 153-165. 